\title{
Sound Level Monitoring at Live Events, Part 1-Live Dynamic Range
}

\author{
ADAM J. HILL, ${ }^{1}$ AES Member, JOHANNES MULDER, ${ }^{2}$ \\ (a.hill@derby.ac.uk) (Jos.Mulder@anu.edu.au) \\ JON BURTON, ${ }^{1}$ AES Member, MARCEL KOK, ${ }^{3}$ AES Student Member AND \\ (j.burton2@derby.ac.uk) \\ (mail@dbcontrol.nl) \\ MICHAEL LAWRENCE ${ }^{4}$ \\ (michael@rationalacoustics.com) \\ ${ }^{1}$ College of Science and Engineering, University of Derby, Derby, DE22 1GB, UK. \\ ${ }^{2}$ College of Arts and Social Sciences, The National University of Australia, Canberra, Australia. \\ ${ }^{3} d$ Bcontrol, Zwaag The Netherlands. \\ ${ }^{4}$ Rational Acoustics, Woodstock, CT, USA.
}

\begin{abstract}
Musical dynamics are often central within pieces of music and are therefore likely to be fundamental to the live event listening experience. While metrics exist in broadcasting and recording to quantify dynamics, such measures work on high-resolution data. Live event sound level monitoring data is typically low-resolution (logged at one second intervals or less), which necessitates bespoke musical dynamics quantification. Live dynamic range (LDR) is presented and validated here to serve this purpose, where measurement data is conditioned to remove song breaks and sound level regulation-imposed adjustments to extract the true musical dynamics from a live performance. Results show consistent objective performance of the algorithm, as tested on synthetic data as well as datasets from previous performances.
\end{abstract}

\section{INTRODUCTION}

Sound level monitoring and management at live events is becoming increasingly important with the ongoing introduction of prescriptive sound level regulations [1]. The motivation of many regulations is twofold: protecting audience members from hearing damage and minimizing annoyance in the local community. This is the first of a trio of papers aimed at developing a more robust understanding of the effect of sound level monitoring practices on regulation compliance as well as the audience experience.

It is well-known that dynamics can be effectively used for musical expression [2]. Following this, one of the more exciting aspects of live music is likely to be the dynamic nature of the reinforced sound. In line with the current emphasis on the "democracy of sound," a situation in which every audience member receives the same excellent listening experience, it is critical to ensure that strong, high-quality sound is delivered to every seat in a venue. Useful quantifiers in this regard include intelligibility, clarity, broadband magnitude response, and dynamic range $[3,4]$. Along these lines, modern audiences have come to expect a strong tactile response, which is directly related to wide dynamic range low-frequency reinforcement [5].
As such it's important to quantify dynamic range in a live music environment. The question is how to best process and analyze the incoming data to give an accurate and reliable indicator of musical dynamics?

This paper details research into a novel musical dynamics metric specifically developed for sound reinforcement applications. The focus is exclusively on the objective development of such a metric in this instance. While a subjectively tuned metric would be ideal, it must be noted that the most notable techniques for analyzing dynamic range in music have been previously found to provide poor correlation between objective and subjective data [6], although further research is required to fully validate this notion.

Research detailed in the proceeding two papers in this series focuses on the impact of sound level regulations on the audience experience at live events. Such an analysis requires a musical dynamics metric to provide a robust and repeatable analysis of recorded data to identify significant objective effects of specific sound level regulations.

A review of relevant literature in the field is presented in Sec. 1, including a justification for a new metric of this variety. Sec. 2 describes the proposed algorithm, while Sec. 3 details verification of the algorithm using synthetic data as well as sound level monitoring data collected by the authors. 
The paper concludes in Sec. 4, including suggestions for further work in this area.

\section{BACKGROUND}

There has been a steady stream of research related to program content dynamics over the past few decades, primarily focused on broadcast media [7-9] and recorded music [6,10-14]. Two previous case studies by the authors are the only known formally published research that analyzes musical dynamics at live events $[15,16]$.

\subsection{Loudness Range}

The International Telecommunications Union (ITU) Recommendation ITU-R BS.1770-4 [7] sets out clear guidelines on how to quantify the loudness of broadcast material. The European Broadcast Union supplemented their recommendation in this area (EBU R128 [8]), which was largely based on the ITU recommendation, with EBU-Tech 3342 , a description of an algorithm to quantify the so-called Loudness Range (LRA) [9]. LRA measures the loudness variation (in loudness units, LU) over a macroscopic time scale. The algorithm is not to be confused with more commonly quoted metrics such as dynamic range or crest factor.

As broadcast loudness levels can't be assumed to follow any conventional statistical distribution, nonparametric statistical analysis is required. In the case of LRA (and most other related metrics), a percentile-based analysis was adopted [9]. LRA is defined as the difference between the $95^{\text {th }}$ and $10^{\text {th }}$ percentiles, where anything below the $10^{\text {th }}$ percentile is considered non-program material (background noise or silence), while anything above the $95^{\text {th }}$ percentile is treated as an anomalous aural event, such as a single gunshot or an explosion in a film.

Critically, LRA operates on a cascaded gating method. First an absolute threshold is applied, always at - 70 LUFS, to remove non-program material in the signal. Second a relative threshold is applied 20 LUFS below the absolute gated loudness level. This removes uncharacteristically quiet sections of a program that would otherwise artificially elevate the LRA reading [9]. The analysis is carried out using a sliding three second analysis window. Test signals along with a full code listing of the algorithm are provided in the EBU technical supplement [9].

\subsection{Dynamic Spread}

LRA operates on a gated percentile range of loudness levels. It can be argued that this may inadvertently overlook key details within the dynamics of program material. This prompted the development of Dynamic Spread (DS) [10]. DS was formulated based on three desirable characteristics, namely that the metric should

1) be unaffected by global gain adjustments,

2) show identical scaling when all individual components are identically scaled, and

3) be based on all analysis frame values.
The DS is generally defined as

$$
D S=\left(\frac{1}{M} \sum_{i=0}^{M-1}\left|V_{d B}(i)-\bar{V}\right|^{p}\right)^{\frac{1}{p}}
$$

where dynamic spread, $D S$, is calculated over $M$ analysis frames, using the absolute difference between the RMS voltage expressed in decibels $\left(V_{d B}\right)$ and the mean $V_{d B}$ value, $\bar{V}$, over all analysis frames. The influence of outliers within the dataset is controlled by the constant, $p$. Higher values result in greater influence of outliers. In the original study [10] it was found that $p=1$ was ideal, corresponding to the mean absolute deviation of the $V_{d B}$ values. DS therefore includes all values within each analysis frame in its characterization of program material dynamics, as opposed to loudness range where dynamics are calculated based on the gated extremes.

\subsection{Higher-Order Statistical Analysis}

As noted in the formulation of DS, it could be problematic if program dynamics are restricted to a straightforward percentile analysis [10]. A solution to this potential issue involves higher order statistical analysis. Such analysis has been carried out by several researchers, all principally focused on musical signals [11-14].

The majority of such research led to a focus on skewness and kurtosis of the histograms of level distribution in particular pieces of music [11-13]. In short skewness indicates any noncentral tendency of a measurement's sound level distribution, while kurtosis indicates the nature of the peak and tails within the sound level distribution data [13]. Such metrics could prove invaluable as it is theoretically possible to arrive at identical values from differently shaped histograms using a percentile range analysis. While it is not yet understood if or how these measures relate to human perception of music (many of these metrics appear to be largely uncorrelated with subjective ratings [6]), from an objective viewpoint care must be taken not to inadvertently discard valuable data, and therefore such analysis should be considered in any robust analysis of program material dynamics.

\subsection{Relevance to Sound Reinforcement}

Each of the aforementioned studies [10-14] focuses on broadcast or recorded material, where it is commonplace to have access to high-resolution data (sampling rates at or above $44.1 \mathrm{kHz}$ ). Considering sound level monitoring at live events, it is common to only have access to low-resolution data (logged at one second intervals or less), making the aforementioned analysis methods unsuitable.

Sound level monitoring packages beyond basic sound level meters operate using equivalent continuous sound pressure level $\left(L_{e q}\right)$ as defined by Eq. (2) for instances where discrete samples are used [17].

$$
L_{e q, T}=10 \log _{10}\left(\frac{1}{T} \sum T_{i} 10^{0.1 L_{e q, T_{i}}}\right)
$$


where $L_{e q}$ is calculated based on the $L_{e q}$ time frame, $T$, the sampling period of the discrete $\mathrm{L}_{\mathrm{eq}}$ calculation, $T_{i}$, and the $L_{e q}$ for each individual sample $L_{e q, T i}$.

In live event applications, it is now standard practice for the base-level $L_{e q}$ measurement time frame to be $1 \mathrm{~s}$ ( $L_{\text {Aeq,1sec }}$ and $L_{C e q, 1 s e c}$ ) [18]. Although it is theoretically possible to process the incoming data at higher resolutions, existing software distills the input data into $L_{e q, 1 \mathrm{sec}}$ points to limit requirements for processing power and memory (it is often a requirement to store tamper-proof log files of sound level data from an event. One-second resolution results in file sizes of a few $\mathrm{MB}$ as opposed to a few GB for higher resolution data).

With this restriction in mind (especially considering that all existing verified sound level monitoring data is lowresolution), any analysis of live event dynamics will be unable to directly implement the previously described techniques. Instead, a bespoke method should draw on best practice from what has come before.

\subsection{Further Challenges}

During a live event, it is common for an $L_{e q}$ limit to be in place. The sound engineer will often have to adjust the overall level (often using the master fader, VCA/DCA faders, individual channel faders, compression, equalization, or a combination of multiple techniques) to ensure compliance with the limit. The process of a sound engineer lowering the level to comply with a limit could artificially increase any measure of dynamics at an event.

For example if a band starts playing with a sound pressure level (SPL) constrained to within $\pm 5 \mathrm{~dB}$, this will give a simplified dynamic range of $10 \mathrm{~dB}$. If the engineer has to reduce the output of the mix by $3 \mathrm{~dB}$, however, a conventional analysis could identify the simplified dynamic range of the music as $13 \mathrm{~dB}$, which is incorrect.

An example of this was observed in previous work in this area, where a case study was conducted on measurement data collected at a large outdoor music festival [15]. On the Saturday of the festival, there were two indie rock acts performing back-to-back. The first act started their performance with an SPL above the local limit ( $\left.96 \mathrm{~dB} L_{A e q, 5 \text { min }}\right)$. This resulted in the sound engineer having to decrease the playback level a few songs into the set. Additionally the act had long breaks between songs, which were included in the musical dynamics analysis. The resulting dynamic range (calculated based on the raw data using L10 - L90, the difference between the $L_{A e q, 1 s e c}$ value exceeded $10 \%$ of the event and $L_{A e q, 1 s e c}$ value exceeded $90 \%$ of the analysis duration) was $18.0 \mathrm{dBA}$.

The following act was similar in musical style to the first act, but this act's engineer complied with the sound level limit for the duration of the performance, and the act had minimal breaks between songs. This resulted in a dynamic range of $7.9 \mathrm{dBA}$. While both acts provided a similar musical listening experience, there was over $10 \mathrm{~dB}$ difference in the calculated dynamic range (Fig. 1). This was due to the adjustments by the first sound engineer as well as the inclusion of breaks between songs in the data
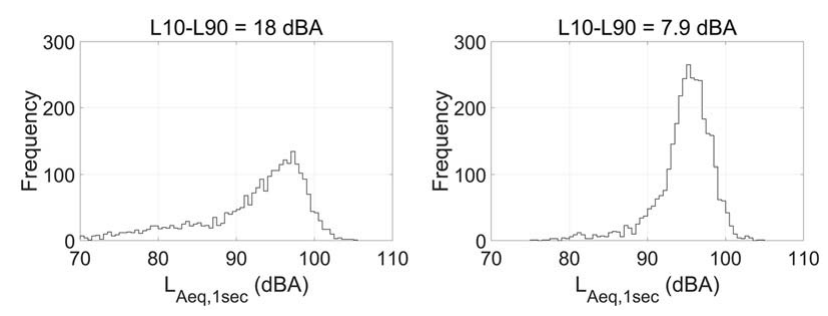

Fig. 1. Example of inaccuracies in musical dynamics due to adjustments for sound level limit compliance (left: first band with long song breaks and necessary overall level adjustments, right: second band with minimal song breaks and no overall level adjustments required). Adapted from [15].

analysis. It is not a true reflection of the dynamic content of the music.

One solution is to analyze dynamic range in short time frames (such as on a song-by-song basis or with a sliding analysis window as in [9]) and take an average across an entire performance. This would require manual tagging of song starts and finishes within a set and could prevent capturing long-term dynamic progression of the music over an entire performance. Another option is to automatically remove level adjustments by the sound engineer (in response to the imposed sound level limit) and song breaks, thus focusing the analysis on an act's musical dynamics.

Of the two options, the latter is more appropriate because it can handle sound level regulation-imposed adjustments at any point in a performance, even within a single song (which is often when the engineer makes adjustments). It's critical that any such engineer-based level adjustments don't impact the musical dynamics calculation but equally important that adjustments by the engineer directly contributing to the musical listening experience of the audience are maintained.

\section{LIVE DYNAMIC RANGE}

Drawing on the EBU's loudness range metric [9], a novel metric targeting sound reinforcement applications was developed, termed live dynamic range (LDR). LDR is based on L3 - L90 (i.e., the difference between the $L_{e q, 1 s e c}$ values exceeded $3 \%$ and $90 \%$ of the analysis duration, respectively). This suppresses any spurious peaks in the data (such as bumps into the measurement microphone) as well as ambient noise between songs (which aligns with background noise in BS 4142 [17]). L3 was chosen to balance the importance of sharp transients in live sound and spurious readings that would skew the results. Initial testing indicated that LDR was inconsistent when using percentiles higher than the $97^{\text {th }}$. This choice sits approximately in the middle of upper percentiles explored in the literature: $99^{\text {th }}$ in [13], 97.7 th $^{\text {in }}[19]$, and $95^{\text {th }}$ in [9].

Prior to the LDR calculation, the measurement data must be conditioned because the data will inherently contain nonprogram information (i.e., data points where there is no musical content). These points must be removed before calculating LDR; otherwise, LDR will be artificially 
inflated. Nonmusical data points are identified using $L_{C e q}$ data (ideally in one second intervals, although longer intervals can be used, if necessary). $\mathrm{L}_{\mathrm{Ceq}}$ data is used because nonmusical content (audience noise, stage banter) generally does not contain significant low-frequency content and will therefore present a clear distinction between periods during a performance for most musical acts. It is critical that any measurement microphone used for LDR monitoring has an appropriate wind screen to avoid excessive low-frequency wind noise.

As with the EBU's loudness range algorithm, thresholding is used to suppress nonmusical content. A mask is generated from an $L_{C e q}$ threshold based on the difference between the root mean square (RMS) of $\mathrm{L}_{\mathrm{Ceq}}$ and the standard deviation over the data set (Eq. (3)). The mask is generated by comparing the threshold to the raw data (with Gaussian smoothing applied over a 30-s interval). Any time periods falling under the threshold are subsequently removed from the data to ensure only musical content is included in the LDR calculation (Eqs. (4) and (5)).

$$
\begin{aligned}
& k=L_{\mathrm{Ceq}, 1 \mathrm{sec}, \mathrm{RMS}}-\sigma_{L_{\mathrm{Ceq}, \mathrm{Isec}}} \\
& M_{L D R}=\left\{\begin{array}{cc}
1 & \text { if } L_{\mathrm{Ceq}, 1 \mathrm{sec}}>k \\
0 & \text { otherwise }
\end{array}\right. \\
& x_{M}=x_{\text {raw }} \odot M_{L D R}
\end{aligned}
$$

where the raw data, $x_{\text {raw }}$, is masked by $M_{L D R}$ which is set using threshold, $k$, based on the RMS and standard deviation of the $L_{C e q, 1 s e c}$ data, $L_{C e q, 1 s e c, R M S}$ and $\sigma_{L C e q, 1 s e c}$, respectively. This results in masked data, $x_{M}$.

After nonmusical content has been removed from the data, the measurements are processed to suppress nonmusical dynamics, which generally stem from the sound engineer adjusting the overall level of the sound system (not individual instruments) to comply with sound level regulations. Such dynamics in the measured data are relatively slow, whereas the musical dynamics are generally rapid in nature. Within the LDR algorithm nonmusical dynamics are considered fluctuations in the equivalent continuous sound pressure level $\left(L_{A e q}\right.$ and $\left.L_{C e q}\right)$ that take greater than 3 min to be fully realized. It must be emphasized that the contributions by the sound engineer are essential to the musical listening experience of the audience. Only level adjustments in relation to the imposed sound level limit should be filtered from the data.

The masked data is processed to remove nonmusical dynamics using a second order Butterworth highpass filter with a cutoff frequency of $1 / 180 \mathrm{~Hz}$ (relating to a period of $3 \mathrm{~min}$ ), noting that the raw data has a sampling rate of 1 Hz. Once $L_{A e q}$ and $L_{C e q}$ data have been passed through the filter, LDR can be calculated using

$$
L D R=L 3\left(x_{M, H P F}\right)-L 90\left(x_{M, H P F}\right),
$$

where live dynamic range, LDR, is calculated as the difference between L3 and L90, the equivalent continuous sound pressure level exceeded $3 \%$ and $90 \%$ of the measurement period, respectively, as applied to the filtered and masked data, $x_{M, H P F}$.

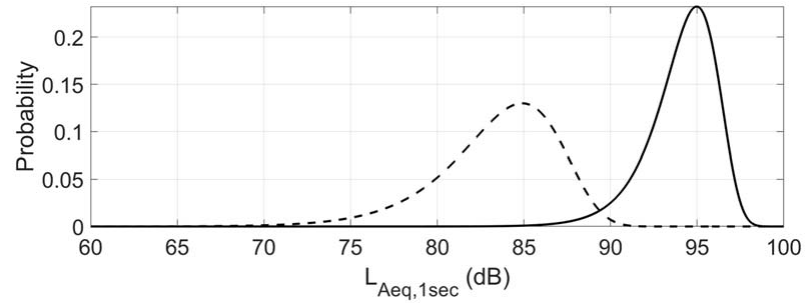

Fig. 2. Probability density functions for the synthesized musical content (solid) and song breaks (dashed)

\section{VERIFICATION}

The live dynamic range (LDR) algorithm must be verified before being used. Again, the focus in this research is on objective performance in the context of musical dynamics in the presence of various sound level regulations. Any consideration of subjective correlation to the LDR metric should be the focus of further work.

\subsection{Synthetic Data}

The LDR algorithm was first verified with synthesized data, using parameters informed by existing data provided by the authors. It was found that both musical content and song break noise spanned a maximum SPL range of 30 $\mathrm{dB}$. A virtual performance of ten 5-min songs was generated with $30 \mathrm{~s}$ between each song. The synthesized data was generated using a pseudorandom algorithm with two Weibull distributions, one for musical content and one for song breaks.

The principal parameters used when defining a Weibull distribution are termed scale, which affects the $x$-axis location of the distribution, and shape (i.e., the Weibull slope), which governs the slope of the resulting distribution [20]. The scales for the musical content and song break noise distributions were set to mean sound pressure levels of 95 $\mathrm{dB}$ and $85 \mathrm{~dB}$, respectively, while the shapes were set to 1,800 and 900 , respectively, divided by the maximum sound pressure range of $30 \mathrm{~dB}$ (Fig. 2).

The virtual sound engineer exhibited a master fader adjustment range of $\pm 2 \mathrm{~dB}$ with a maximum adjustment reached after $20 \mathrm{~min}$. For the simulated data, the engineer's adjustments followed a sinusoidal pattern. $L_{\mathrm{Ceq}}$ data were defined as $10 \mathrm{~dB}$ above the synthesized $L_{A e q}$ data. As LDR is effectively blind to absolute sound pressure level this arbitrary choice is inconsequential.

First, the mask ( $M_{L D R}$ in Eq. (4)) was generated to remove nonmusical content (audience noise and stage banter during song breaks) from the data. As described in Sec. 2, this was carried out with $L_{C e q}$ data (Fig. 3).

The masked data ( $x_{M}$ in Eq. 5) was passed through a highpass filter to remove the engineer-related dynamics, resulting in data consisting only of musical content, $x_{M, H P F}$ (Fig. 4). Note that the data was zero padded by 12,000 samples, with all inserted samples set to the calculated threshold, $k$, at the beginning of the data set to avoid distortions at the start of the $L_{e q, 1 s e c}$ filtered data due to the filter's impulse response. 


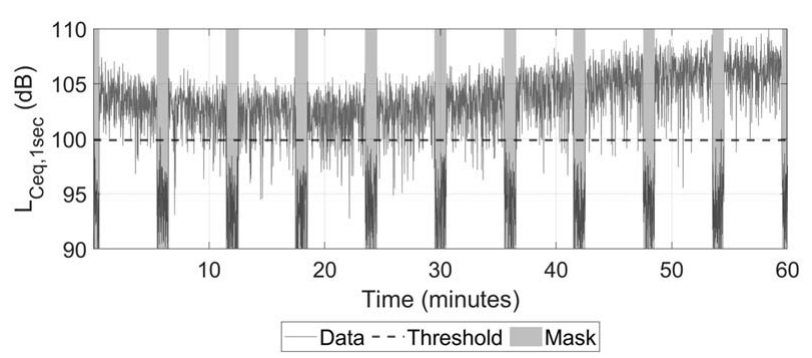

Fig. 3. Example synthesized $L_{C e q}$ data $\left(x_{\text {raw }}\right)$, musical content threshold $(k)$ and the resulting nonmusical content mask $\left(M_{L D R}\right)$ as part of the live dynamic range (LDR) algorithm.

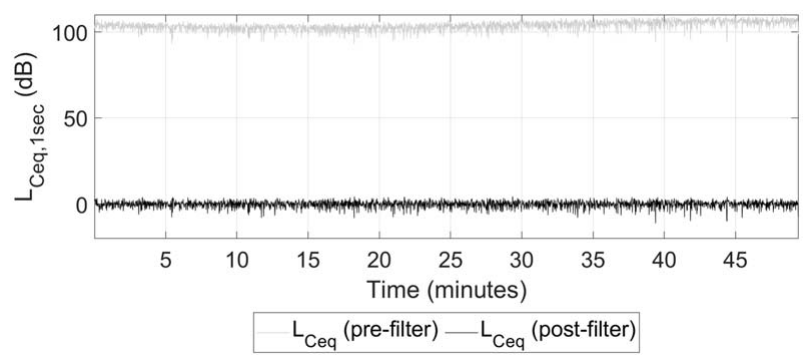

Fig. 4. Example synthesized $L_{C e q}$ with nonmusical measurement points removed, pre-filtering ( $x_{M}$, grey) and post-filtering $\left(x_{M, H P F}\right.$, black $)$.

Table 1. Synthesized variable allowable ranges during randomized testing

\begin{tabular}{ll}
\hline \hline Parameter & Allowable range \\
\hline SPL range (songs) & $10-50 \mathrm{~dB}$ \\
SPL range (audience) & $10-30 \mathrm{~dB}$ \\
Number of songs & $5-15$ songs \\
Minutes between songs & $0-1 \mathrm{~min}$ \\
Minutes per song & $3-8 \mathrm{~min}$ \\
Mean SPL (songs) & $85-105 \mathrm{~dB}$ \\
Mean SPL (audience) & $60-80 \mathrm{~dB}$ \\
Master fader adjustment range & $\pm 0-3 \mathrm{~dB}$ \\
Master fader rate of adjustment & $0-1 \mathrm{mHz}$ \\
\hline
\end{tabular}

SPL $=$ sound pressure level.

The filtered data shows the removal of low-frequency fluctuations in SPL as well as the DC offset, which represents the mean $L_{C e q}$. For this particular set of synthesized data, LDR was calculated to be $5.52 \mathrm{~dB}$ (A- and C-weighted LDR are identical here as the $L_{C e q}$ data were directly generated from the $L_{\text {Aeq }}$ data).

As the LDR algorithm operates between the $10^{\text {th }}$ and $97^{\text {th }}$ percentiles of $L_{e q}$ data, the LDR with the Weibull distributed data should result in a value of $5.50 \mathrm{~dB}$. The calculated LDR exhibited a difference of $0.02 \mathrm{~dB}(0.36 \%$ error $)$ to the expected value.

To further validate the LDR algorithm using synthesized data, different combinations of settings were tested. Ten thousand configurations were explored in which the settings for the synthesis variables were randomized according to Table 1. True versus calculated LDR values are analyzed in Fig. 5.

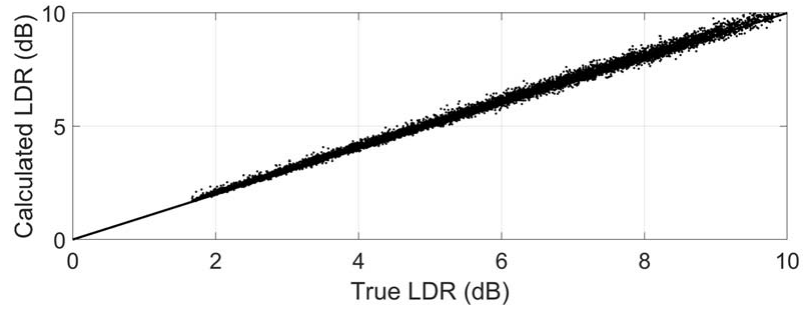

Fig. 5 Comparison of the calculated vs. true LDR values for 10,000 randomly generated synthesis configurations. LDR $=$ live dynamic range.

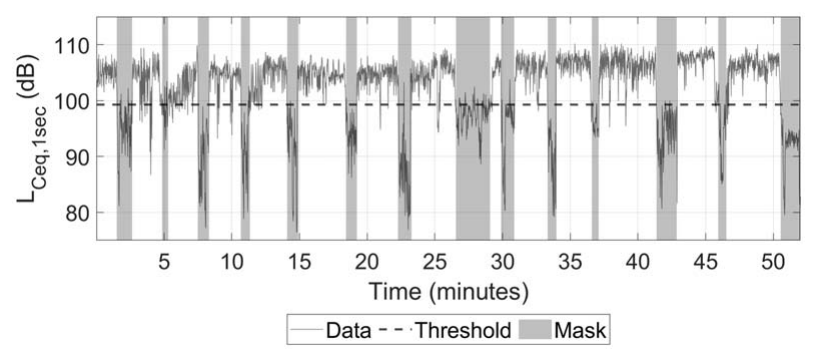

Fig. 6 Example nonmusical mask generation for real-world data collected at a music festival in 2019.

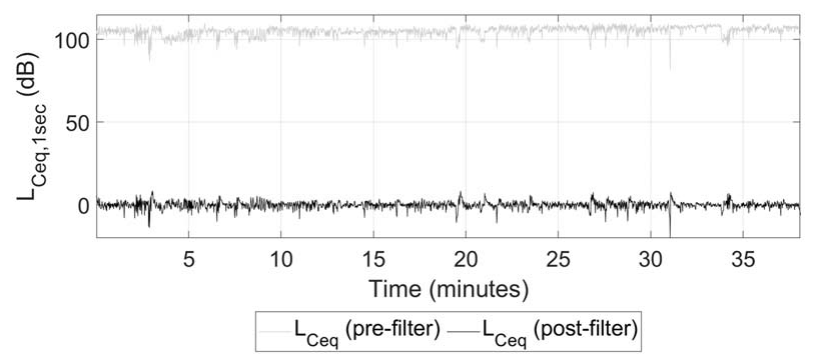

Fig. 7 Example musical data filtering for real-world data collected at a music festival in 2019 .

The algorithm accurately calculates LDR, with a root mean squared error of $0.025 \mathrm{~dB}$. The most significant error is observed for trials where there is substantial overlap between the mean song level and audience level, thus preventing the algorithm from removing all song break data. The maximum LDR error within these tests was $1.04 \mathrm{~dB}$ (corresponding to an error of $11.3 \%$ ).

\subsection{Single Music Festival $\left(L_{e q, 1 s e c}\right)$}

The LDR algorithm was next tested on real-world data taken at a music festival in 2019 [15]. A single band's performance data are shown in Fig. 6, highlighting the application of the nonmusical content mask, and in Fig. 7, revealing the data after highpass filtering. In this instance the $\mathrm{A}$ - and $\mathrm{C}$-weighted LDR values were calculated as 7.57 $\mathrm{dBA}$ and $6.44 \mathrm{dBC}$, respectively (the act was a three-piece funk band).

For A- and C-weighted LDR, the observed ranges over all 23 acts at the festival were 5.50-12.03 dBA and 5.09$14.15 \mathrm{dBC}$, respectively. As the LDR algorithm ignores the 

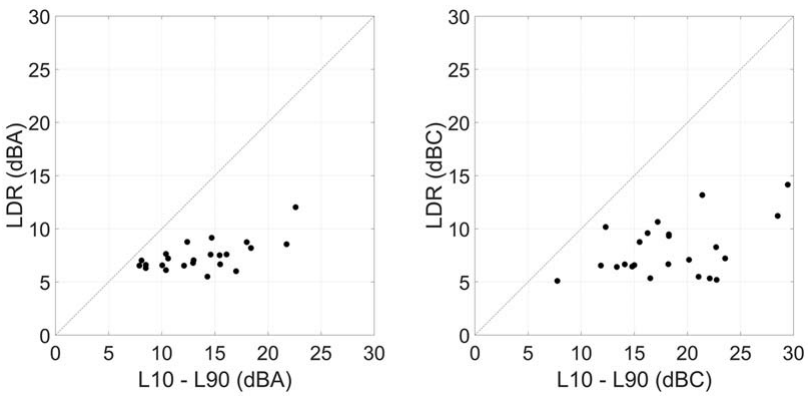

Fig. 8 Comparison of original live dynamic range calculation (L10 - L90) and the new LDR algorithm using data from the 2019 music festival. $\mathrm{LDR}=$ live dynamic range.

top $3 \%$ and bottom $10 \%$ of the $\mathrm{L}_{\mathrm{eq}}$ range, these results indicate that all acts generally stayed within a controlled operating range of sound levels, with dynamics expressed in short time intervals. This is consistent with previous findings $[15,16]$.

As a final examination of the improved LDR algorithm (as compared with more basic methods outlined in the previous study [15]), each act from the 2019 festival was reanalyzed with the LDR algorithm and directly compared with the original approach (which had no mechanism to exclude song breaks and sound level limit-related adjustments). A comparison between the two approaches is provided in Fig. 8, with the L10 - L90 and LDR histograms reproduced in Figs. 9 and 10. For completeness, the fully conditioned LDR histograms, including higher-order statistics, as described in Sec. 1.3, are provided in Fig. 11. The missing plots in Figs. 9-11 indicate cancelled performances due to adverse weather conditions at the festival [15].

The comparison between the two data sets in Figs. 9 and 10 highlights the importance of removing nonmusical data before analyzing music dynamics from a live event. In many cases, there is a significant (greater than $10 \mathrm{~dB}$ ) left tail to the histograms due to the song break data being included in the analysis. This skews the dynamics analysis, giving an inaccurate assessment of reality.

The L10 - L90 calculations from the original case study [15] show musical dynamics approaching $30 \mathrm{dBC}$ in some cases. After the LDR algorithm was applied the maximum level of musical dynamics observed is just above $14 \mathrm{dBC}$. The $\mathrm{C}$-weighted comparisons are more pronounced than the A-weighted comparisons because the song breaks typically have minimal low-frequency content, creating an artificially inflated dynamic range of the raw data, which bears no significance to the musical content.

The analysis presented in Fig. 11 allows for inspection of higher-order statistics, namely skewness $\left(\gamma_{1}\right.$, where nearly all analyzed performances show a positive value indicating a longer tail in the positive direction-a tendency for amplitude peaks) and excess kurtosis $\left(\gamma_{2}\right.$, where most values are clustered around zero, indicating a tendency towards a normal distribution. Positive values highlight a sharp peak and more significant tails, whereas negative values indicate the opposite).

Upon examining these statistics, it was determined that LDR was adequate as an independent measure of live musical dynamics, without the need for higher-order statistical modifiers. In all observed instances, LDR adequately captured the dynamic spread of the data.

\subsection{Single Music Festival $\left(L_{e q, 1 m i n}\right)$}

The LDR algorithm's performance must be analyzed when $L_{e q, 1 s e c}$ data aren't available. While not ideal, $L_{e q, 1 \mathrm{~min}}$ data are often all that is available in certain sound level measurement $\log$ files [16]. $L_{e q, I \min }$ data aren't ideal for LDR calculations as they lack the appropriate time resolution to track musical dynamics. It is also likely that song breaks will be overlooked in the analysis unless they are on the order of 1 min or longer.

As a starting point, the same data displayed in Figs. 6 and 7 were inspected using $L_{e q, 1 \min }$ data rather than the original $L_{e q, 1 s e c}$ data (Figs. 12 and 13). The $L_{e q, 1 \min }$ LDR was 5.22

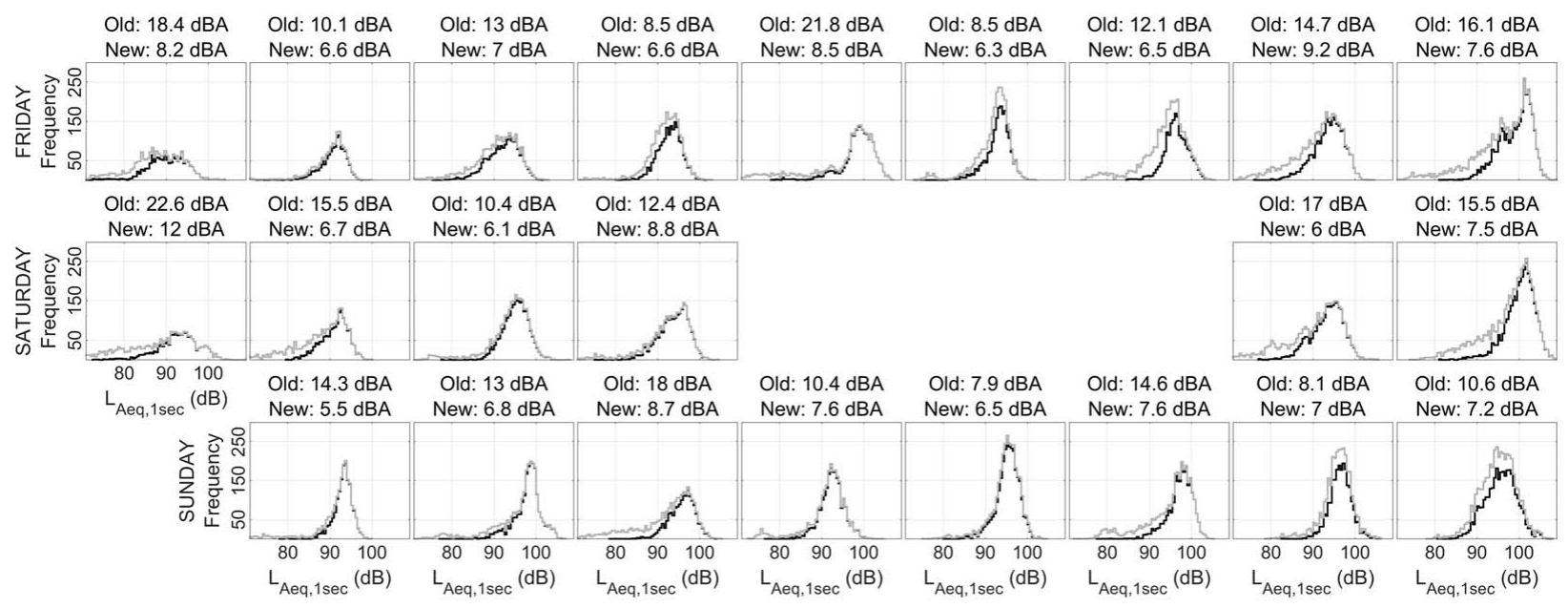

Fig. 9 L10 - L90 (old) and LDR (new) A-weighted histogram analysis for each act from the 3-day festival in 2019 (grey traces = histogram of raw data, black traces $=$ histogram after removal of song breaks). LDR $=$ live dynamic range. 


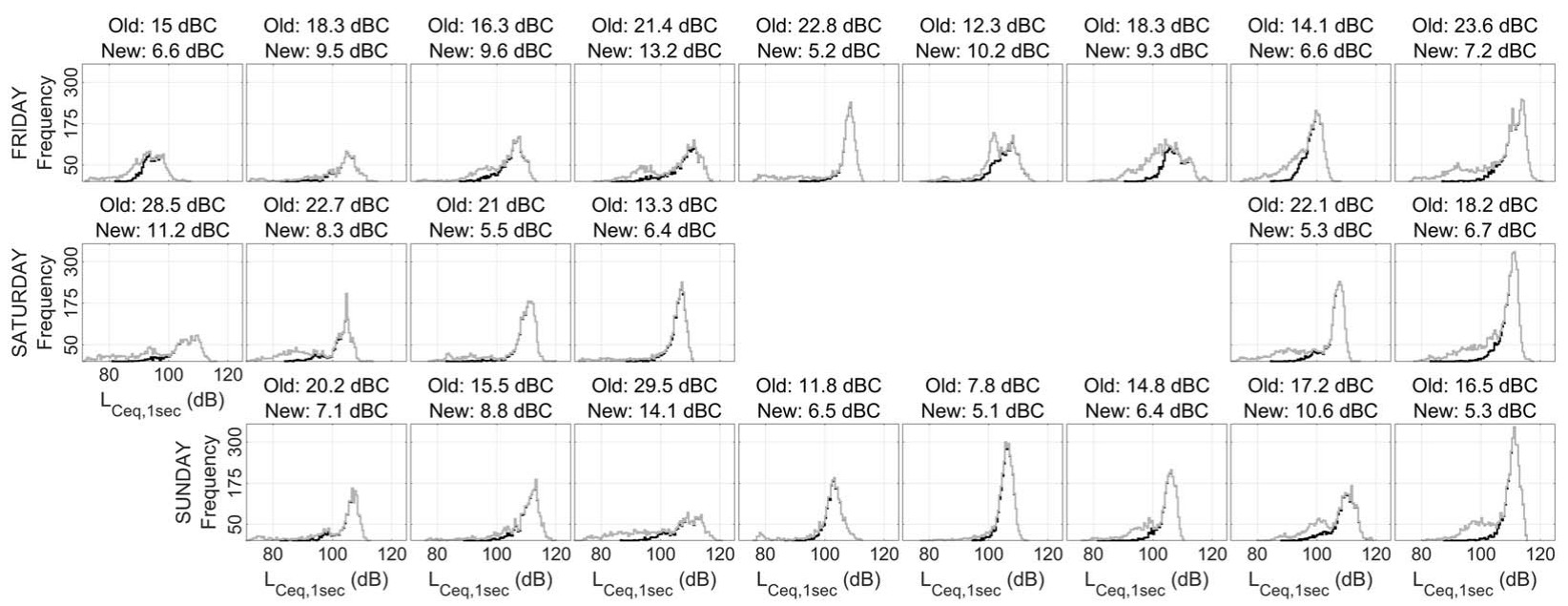

Fig. 10. L10 - L90 (old) and LDR (new) C-weighted histogram analysis for each act from the 3-day festival in 2019 (grey traces = histogram of raw data, black traces $=$ histogram after removal of song breaks). LDR $=$ live dynamic range.

$\mathrm{dBA}$ and $3.70 \mathrm{dBC}$ (the $L_{e q, 1 s e c} \mathrm{LDR}$ was $7.57 \mathrm{dBA}$ and $6.44 \mathrm{dBC})$, considerable underestimates.

To provide a clearer picture of LDR using 1-min data, a comparison can be performed between 1-min and 1-s data over all 23 acts from the 2019 dataset (Fig. 14). The Aand C-weighted LDR comparison root mean squared errors were $2.10 \mathrm{dBA}$ and $2.66 \mathrm{dBC}$, respectively. This confirms the notion that the 1-min data lack much of the musical dynamics captured in the 1-s data.

While this shows a somewhat satisfactory match to the expected 1:1 input-to-output relationship, the specific data show considerable inaccuracies. It is clear from this analysis that 1-min data should only be used if absolutely necessary; 1 -s $L_{e q}$ data are ideally suited to fully capture musical dynamics. Informal testing of intermediate sampling rates was carried out, giving some indication of accurate results when taking a sample every $3 \mathrm{~s}$, but further work is necessary to precisely determine the actual lower sampling limit for LDR.

\subsection{Multiple Outdoor Events}

Measurement data spanning 137 outdoor music events in Europe from 2019 courtesy of one of the authors were analyzed using the LDR algorithm to further inspect the handling of 1-min data (both $L_{e q, 1 s e c}$ and $L_{e q, I m i n}$ were available here). The events ranged from electronic dance music (EDM) festivals to softer contemporary rock performances.

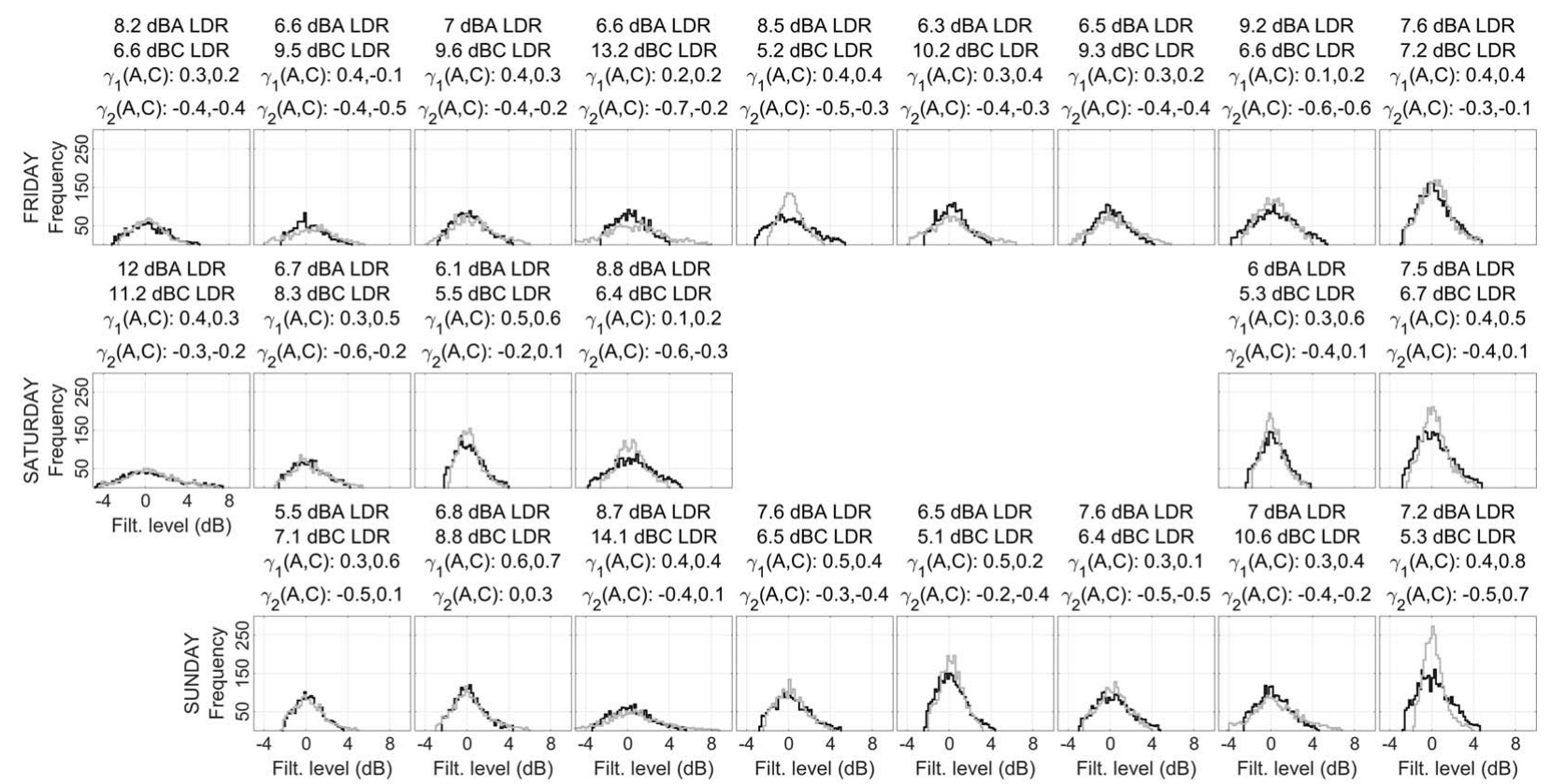

Fig. 11. Fully conditioned LDR histogram analysis for each act from the 3-day festival in 2019 (black traces = A-weighted, grey traces $=\mathrm{C}$-weighted, $\gamma_{1}=$ skewness, $\gamma_{2}=$ excess kurtosis). $\mathrm{LDR}=$ live dynamic range. 


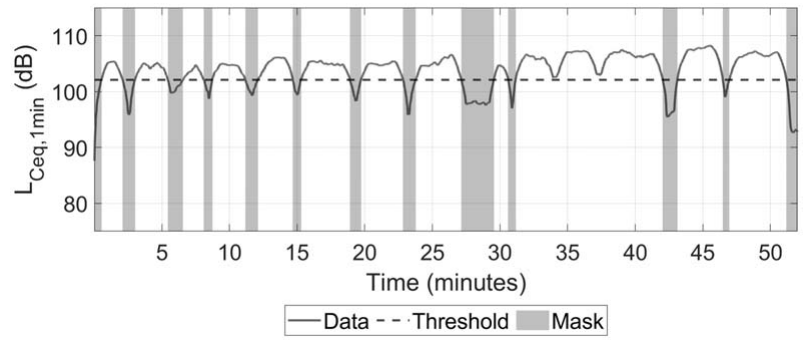

Fig. 12. Example nonmusical mask generation for real-world data collected at a music festival in 2019.

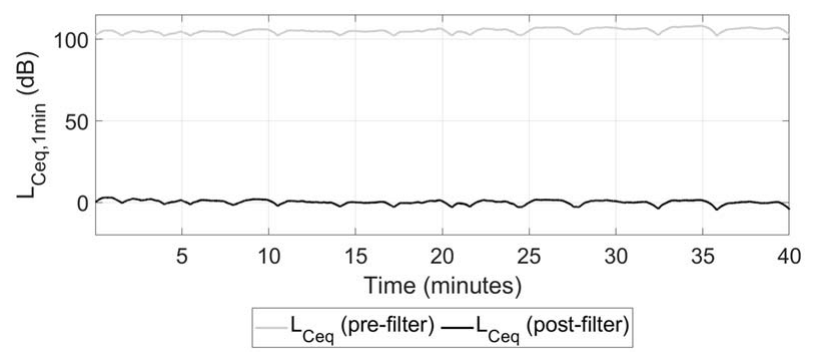

Fig. 13. Example musical data filtering for real-world data collected at a music festival in 2019.
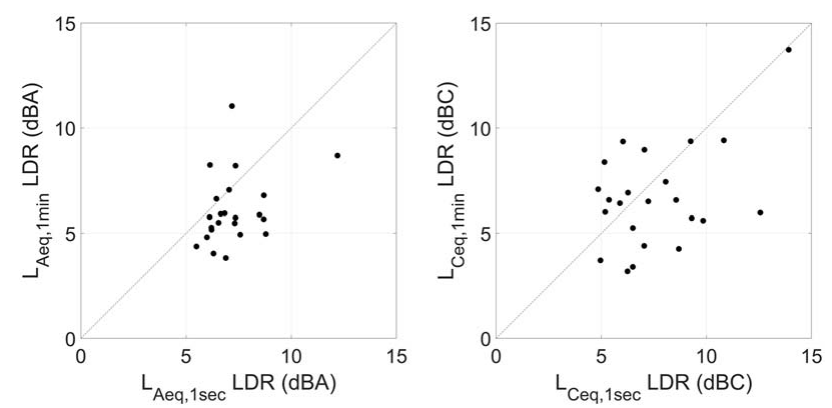

Fig. 14. Comparison $L_{e q, 1 s e c}$ data (horizontal) and $L_{e q}, 1$ min $L D R$ calculations over 23 acts within 2019 music festival data set for Aweighted (left) and $\mathrm{C}$-weighted (right) data. $\mathrm{LDR}=$ live dynamic range.

The observed range of $L_{e q, 1 s e c} \mathrm{~A}$ - and C-weighted LDR over all events was 2.48-8.82 dBA and 3.99-15.18 dBC, respectively. The observed range of the $L_{e q, I m i n} \mathrm{~A}-$ and C-weighted LDR over all events was 2.92-8.46 dBA and 3.35-7.63 dBC, respectively. A comparison between LDR derived from $L_{e q, 1 s e c}$ and $L_{e q, 1 m i n}$ data is given in Fig. 15. The 1-s to 1-min LDR calculations show similar trends to the 2019 music festival data from Sec. 3.3, in this case with A- and C-weighted root mean squared errors of $1.18 \mathrm{dBA}$ and $5.42 \mathrm{dBC}$, respectively, with a tendency to significantly underestimate LDR with the C-weighted data.

Overall the LDR algorithm, when used with $L_{e q, 1 s e c}$ data, has been verified to provide an accurate and repeatable method for analyzing the isolated dynamic range of the musical content at a live event. Values are expected to remain below $20 \mathrm{~dB}$, where an LDR above approximately $8 \mathrm{~dB}$ can be considered to represent a performance with strong musical dynamics, although further work is required
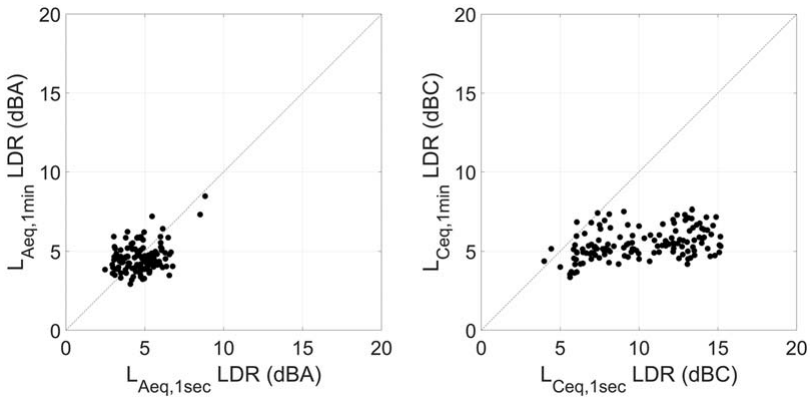

Fig. 15. Comparison $L_{e q(1 \mathrm{sec})}$ data (horizontal) and $L_{e q(1 \mathrm{~min})} \mathrm{LDR}$ calculations over all 137 events for A-weighted (left) and Cweighted (right) data. LDR = live dynamic range.

to refine this threshold. The use of $L_{e q, 1 \min }$ data are not recommended, as this does not provide a robust metric for musical dynamics at live events.

\subsection{Comparison to Other Delivery Formats}

As LDR departs from conventional dynamic quantification algorithms, due to the suppression of song break data and sound level limit-imposed adjustments to the level by the engineer, it is instructive to analyze content delivered through the mediums covered by other algorithms [6-14], namely recorded and broadcast music.

To facilitate this, ten example recordings were obtained from each of the following four categories:

1. Radio (live), live music performed at and broadcast from a commercial radio station.

2. Radio (studio), recorded music played back and broadcast from a commercial radio station.

3. Live album, commercial release of a live performance recording.

4. Studio album, commercial release of a collection of studio recordings.

The radio examples were obtained from the Online Archive, all from past BBC Radio 6 Music shows due to the station's regular live music broadcasts. The album examples were obtained from the primary author's collection, all at CD quality (44.1 kHz, 16-bit depth).

Initial analysis gave LDR values around $20 \mathrm{~dB}$ (A- and $\mathrm{C}$-weighted). The lack of background noise was identified as the cause of this. Datasets A, B, and C show background noise levels around $50 \mathrm{dBA}$ and $60 \mathrm{dBC}$. Because LDR was developed to operate with such noise, it was necessary to impose artificial background noise on the other example recordings to permit a fair comparison. In this instance, uniformly distributed noise was added spanning 25-50 dBA and 35-60 dBC. All examples were analyzed alongside the live event datasets (Fig. 16).

Broadcast music exhibits consistently lower LDR compared with the other examples. The consistency of radio's LDR is expected as all examples were taken from the same station, hence all broadcasts had the same compression applied. The studio examples exhibit higher LDR in some 


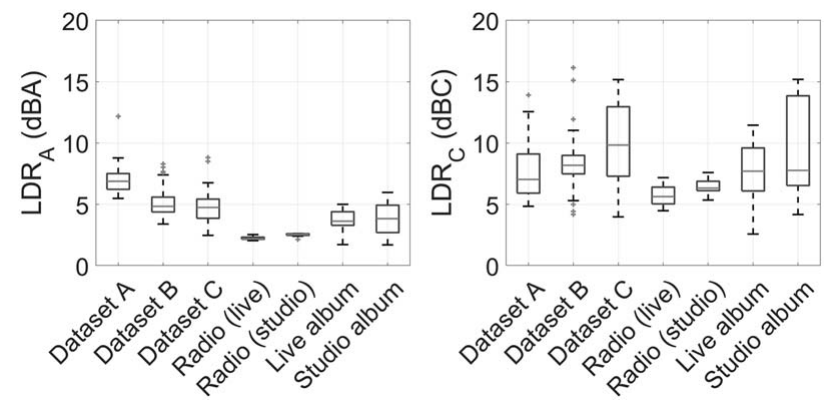

Fig. 16. LDR comparison between live events (Datasets A, B, C), radio- and studio-based music recordings/performances. $\mathrm{LDR}=$ live dynamic range.

cases but lower in others, indicating the wide variety of mastering techniques between studios. The studio examples show that some albums have wide $\mathrm{C}$-weighted dynamics, as compared to any other medium.

The median LDR values for A- and C-weighting are generally in line with live events, however, indicating that modern day live performances are achieving similar listening experiences to what's delivered by an album (aside from the visual, tactile, and social/emotional elements of a live performance).

\section{CONCLUSIONS AND FURTHER WORK}

Recent developments in the area of live event sound level monitoring and management have necessitated a closer inspection of the impact that sound level regulations have on the musical listening experience of an audience. Although the impact on overall sound pressure level can be easily determined from measurement data, the impact on musical dynamics isn't as straightforward to ascertain.

While a number of well-established measures exist within the broadcast and recorded music industries, these operate on high-resolution audio, whereas data logged at live events are generally low-resolution, with a sampling rate of $1 \mathrm{~Hz}$, at best. This necessitated the development of the novel LDR metric.

The LDR algorithm identifies and removes any song break data and then filters the resulting data to remove engineer-related sound level adjustments (which are not directly relevant to the musical dynamics). This leaves the core musical dynamics content within the data, which can be quantified as L3 - L90.

Further work on LDR requires a thorough investigation into the subjective relevance of the metric, specifically determining the just noticeable difference in musical dynamics in a live event setting in order to calibrate a subjective scale for LDR values. Additionally, the LDR algorithm could be explored for implementation in real-time sound level monitoring applications by implementing a sliding analysis window alongside a cumulative analysis, with appropriate initial conditions for the masking threshold to provide sound engineers with additional tools in shaping dynamic and regulation-compliant (safe) mixes. To encourage wider participation in further research, the source code for the LDR algorithm is available at www.soundlevelmanagement.com.

The objective analysis of LDR feeds the investigations in Parts 2 and 3 of this paper series, whereby various sound level regulations are inspected using existing captured data to determine their measurable effect(s) on the objective musical listening experience of the audience. This knowledge in turn is used to develop robust methods for sound level monitoring and management in order to deliver the best possible listing experience at live events while complying with sound level regulations.

\section{REFERENCES}

[1] E. F. Beach, J. Mulder, I O'Brien, and R. Cowan, "Overview of Laws and Regulations Aimed at Protecting the Hearing of Patrons Within Entertainment Venues," Eur. J. Public Health, vol. 31, no. 1, pp. 227-233 (2020 Oct.). https://doi.org/10.1093/eurpub/ckaa149.

[2] R. Arnheim, "Perceptual Dynamics in Musical Expression," Music. Quart., vol. 70, no. 3, pp. 295-309 (1984 Jul.). https://doi.org/10.1093/mq/LXX.3.295.

[3] A. J. Hill (chairman and editor), Understanding and Managing Sound Exposure and Noise Pollution at Outdoor Events. Audio Engineering Society Technical Document AESTD1007.1.20-05 (2020 May).

[4] B. Støfringsdal, "Expected Sound Levels at Concert Venues for Amplified Music," in Proceedings of the Institute of Acoustics Conference on Auditorium Acoustics, vol. 40, pt. 3 (Hamburg, Germany) (2018 Oct.).

[5] A. J. Hill, M. O. J. Hawksford, A. P. Rosenthal, and G. Gand, "Subwoofer Positioning, Orientation and Calibration for Large-Scale Sound Reinforcement," presented at the 128th Convention of the Audio Engineering Society (2010 May), paper 7971.

[6] J. Boley, C. Danner, and M. Lester, "Measuring Dynamics: Comparing and Contrasting Algorithms for the Computation of Dynamic Range," presented at the 129th Convention of the Audio Engineering Society (2010 Nov.), paper 8178 .

[7] International Telecommunication Union, "Algorithms to Measure Audio Programme Loudness and TruePeak Audio level," ITU-R BS.1770-4 (2015).

[8] European Broadcasting Union, "Loudness Normalisation and Permitted Maximum Level of Audio Signals," EBU R128 (2014).

[9] European Broadcasting Union, "Loudness Range: A Descriptor to Supplement Loudness Normalisation in Accordance with EBU R 128,” EBU Tech. Doc. 3342 (2016 Jan.).

[10] E. Vickers, "Automatic Long-Term Loudness and Dynamics Matching," presented at the 111th Convention of the Audio Engineering Society (2010 Nov.), paper 5495.

[11] M. Everett, The Temporal and Spectral Characteristics of Recorded Music, Ph.D. Thesis, Coventry Polytechnic University, Coventry, UK (1988 Oct.). 
[12] E. Benjamin. "Characteristics of Musical Signals," presented at the 97th Convention of the Audio Engineering Society (1994 Nov.), paper 3914.

[13] M. Mijic, D. Masovic, D. Sumarac Pavlovic, and M. Petrovic, "Statistical Properties of Music Signals," presented at the 126th Convention of the Audio Engineering Society (2009 May), paper 7702.

[14] M. Paraskevas and J. Mourjopoulos. "A Statistical Study of the Variability and Features of Audio Signals: Some Preliminary Results," presented at the 100th Convention of the Audio Engineering Society (1996 May), paper 4256.

[15] A. J. Hill, J. Mulder, M. Kok, J. Burton, A. Kociper, and A. Berrios, "A Case Study on Sound Level Monitoring and Management at Large-Scale Music Festivals," in Proceedings of the Institute of Acoustics Conference on Reproduced Sound (Bristol, UK) (2019).

[16] A.J. Hill and J. Burton, "A Case Study on the Impact Live Event Sound Level Regulations Have on Sound
Engineering Practice," in Proceedings of the Institute of Acoustics Conference on Reproduced Sound (online) (2020 Nov.). https://doi.org/10.25144/13382.

[17] J. Navne, "Sound Level Measurements Made Eazy: 10EaZy - Intuitive SPL Monitoring for Live Sound Events," in Proceedings of the Audio Engineering Society 58th International Conference: Music Induced Hearing Disorders (Aalborg, Denmark) (2015).

[18] R. Tollerton, "pfpf: An Experimental Estimator of Dynamic Range in Music," http://audiamorous. blogspot.com/2008/01/pfpf-experimental-estimator-ofdynamic_13.html (accessed Mar. 16, 2021).

[19] British Standards Institute, "Methods for Rating and Assessing Industrial and Commercial Sound," BS 4142:2014+A1:2019 (2019).

[20] S. N. Luko, "A Review of the Weibull Distribution and Selected Engineering Applications," SAE Trans., vol. 108, no. 2, pp. 398-412 (1999 Jan.). http://dx.doi.org/10.4271/1999-01-2859. 
THE AUTHORS

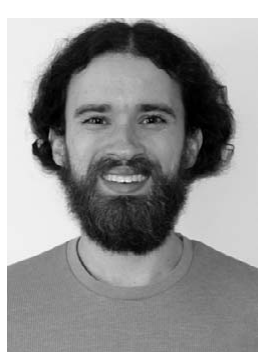

Adam J. Hill

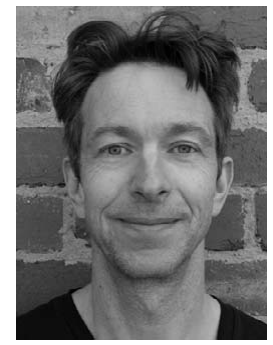

Johannes Mulder

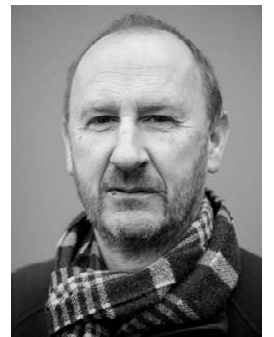

Jon Burton

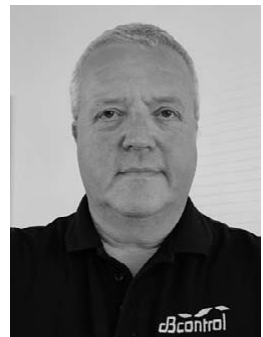

Marcel Kok

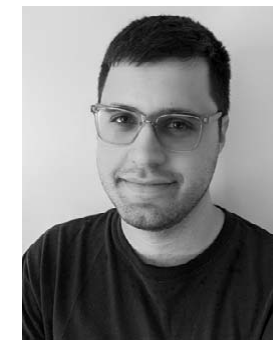

Michael Lawrence
Adam Hill is currently an Associate Professor of Electroacoustics at the University of Derby where he runs the MSc Audio Engineering program. He received a Ph.D. from the University of Essex, an M.Sc. in Acoustics and Music Technology from the University of Edinburgh, and a B.S.E. in Electrical Engineering from Miami University. His research generally focuses on analysis, modeling, and wide-area spatiotemporal control of low-frequency sound reproduction and reinforcement. Adam works seasonally as a live sound engineer for Gand Concert Sound, where he has designed and operated sound systems for over one thousand artists. He is chair of the AES Technical Committee on Acoustics and Sound Reinforcement, a member of the Electro-Acoustics Group Committee within the Institute of Acoustics, and a member of the WHO technical working group for development of the Global Standard for Safe Listening Venues. Adam was awarded the IoA Young Persons' Award for Innovation in Acoustical Engineering in 2019. He is a chartered engineer and member of the AES, IOA, IEEE, and IET.

Johannes (Jos) Mulder is a lecturer in Music Technology at the School of Music, Australian National University. His interdisciplinary research covers different aspects of live sound, including the management and monitoring of sound levels in music venues and the history of live sound practice and technology. Prior to his academic career he worked as a free-lance live sound engineer for 15 years, after studying recording engineering (Tonmeister) at the Royal Conservatoire in The Hague (NL). He has a Master's in Art Management and Administration (2007, University Utrecht) and a PhD (2013, University of Technology, Sydney). He is a Fellow of the Higher Education Academy. Jos is a member of the WHO technical working group for development of the Global Standard for Safe Listening Venues.

Jon Burton is a live sound engineer with many years of touring experience and is currently a senior lecturer at the University of Derby. Jon has toured with acts as varied as Radiohead, Stereophonics, Lulu, and for the last 15 years, The Prodigy. Jon has worked at the highest level for over 30 years. Along with live sound, Jon has worked in TV and radio, on such shows as "Later with Jools." Jon is also a partner in the Laundry Rooms studio complex in Sheffield, which he built from scratch with fellow engineer Dave Hadley. Jon regularly lectures at universities and colleges in the UK and abroad. Having no prior formal education in sound, in 2017 Jon completed an MSc in Music Technology from York University and become a Fellow of the Higher Education Academy. Jon is a part time writer for Sound on Sound magazine and contributes to online education site Soul Sound.

Marcel Kok lives in the Netherlands and is CEO of dBcontrol. The main activity for $\mathrm{dBcontrol}$ is sound level measurements and control at live festivals, followed by consultancy and research on sound levels. Nowadays the market focus is on large outdoor dance events in the Netherlands and Belgium, and there's also dBcontrol Norway in Trondheim. Marcel has a degree in Electrical Engineering at the University of Twente, Netherlands, and has completed an MBA study. His work experience is in the field of acoustic consultancy. Sound engineering has been his hobby since 1982, and as a freelance FOH engineer, he has seen many small venues around Europe. Besides working at $\mathrm{dBcontrol}$, Marcel is a Ph.D. student at Ghent University in Belgium. The topic for his research is the use of $\mathrm{dB}(\mathrm{C})$ as an indicator for the low-frequency annoyance at large outdoor dance festivals. Marcel is a member of the WHO technical working group for development of the Global Standard for Safe Listening Venues.

Michael Lawrence's love affair with professional audio began at age 14, when he was left unattended in his school's auditorium. After graduating from Berklee College of Music (Boston, MA, USA) he toured the country as a sound engineer for various artists before developing a focus on sound system measurement, analysis, and optimization. In 2019 he was adopted by Rational Acoustics, where he works feverishly on the SPL feature set for the Smaart audio analyzer in exchange for a steady diet of tacos and graham crackers. In his free time he enjoys designing and tuning sound systems, which his loved ones do not consider a leisure activity, but they have agreed to disagree. 